\title{
ANAIS DA VIII JORNADA DE FISIOTERAPIA DA CHRISFAPI
}

Realização:

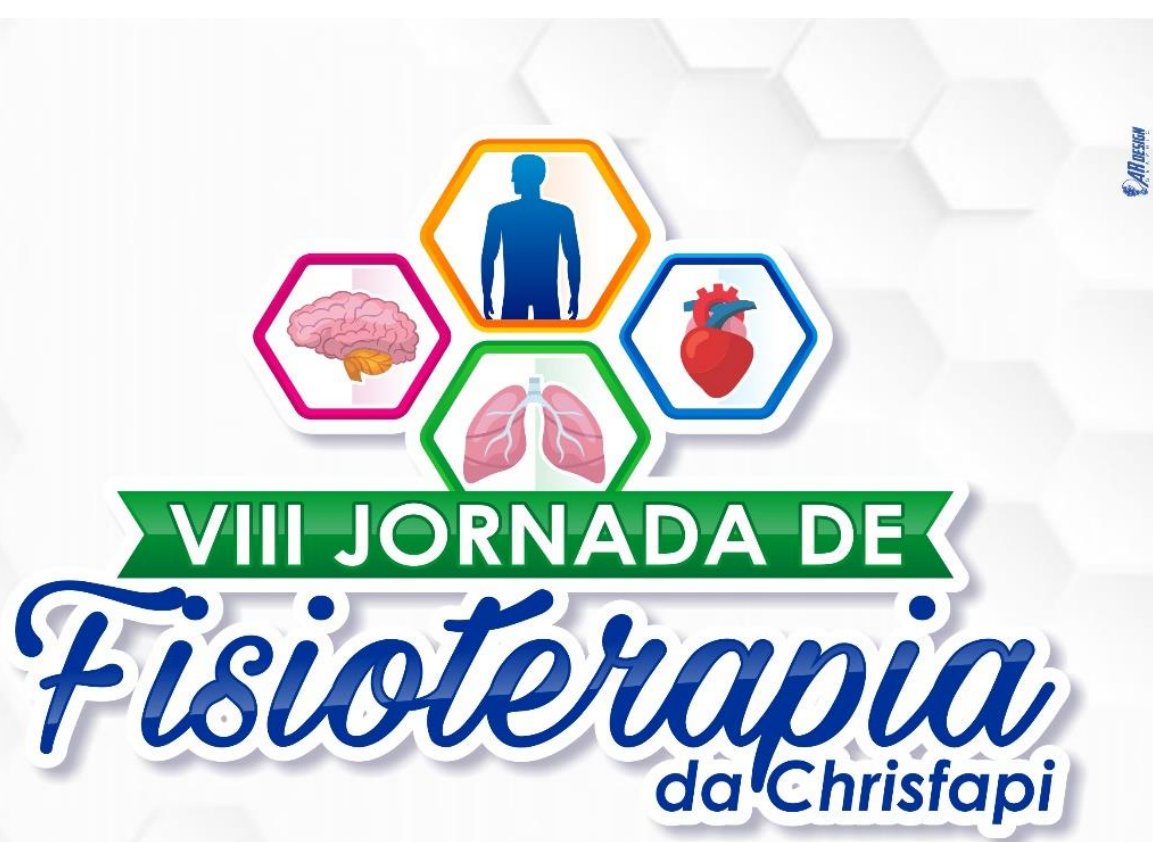

"As Múltiplas Habilidades da Profissão"

Apoio:

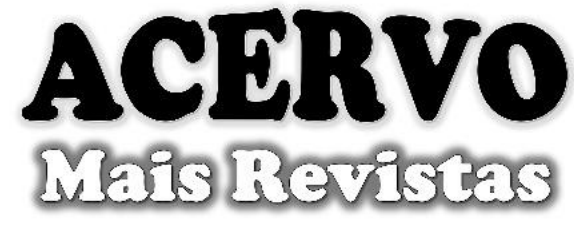

As publicações mais rápidas do país!

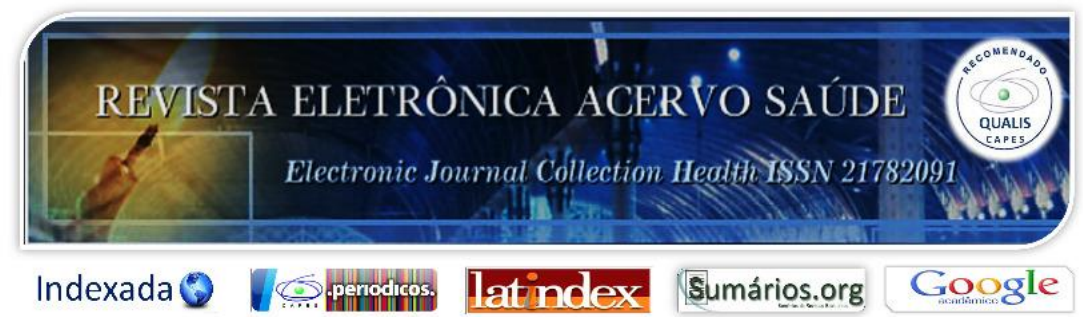


ANAIS DA VIII JORNADA DE FISIOTERAPIA DA CHRISFAPI, 2019;02-38

PRESIDENTE DOCENTE:

Mayane Carneiro Alves

PRESIDENTE DISCENTE:

Dionatan Chistophe Alves de Paula

\section{ORGANIZADORES DO EVENTO}

Alcides Laécio do Nascimento

Amanda Maria Brito da Silva

Ana Clara Freitas Brito Silva

Ana Clara Soares Leite

Beatriz Pereira Melo

Carla Maysla Oliveira Brito

Dionatan Chistophe Alves de Paula

Francisco Márcio Lima Alves

Geicielly Karine de Sousa Santos

Geórgia Araújo Aguiar

Igor Cardoso Araújo

Jéssica Luana Pimentel Silva

Luís Gusthavo Noronha Sousa

Lyandra Ellen Bezerra Meneses

Marcelo Andrade Ribeiro

Maria de Fatima Martins Nascimento

Mauro Cezar Araujo da Silva Junior

Patricia Cardoso Magalhães Medeiros

Raniely dos Santos da Silva

Sabrina Melo Araujo

Sanny Maria Ferreira da Silva

Shirley Pontes da Silva

Taiane Oliveira Pereira 
ANAIS DA VIII JORNADA DE FISIOTERAPIA DA CHRISFAPI, 2019;02-38

\title{
COMISSÃO CIENTÍFICA
}

COORDENADOR E REVISOR CIENTÍFICO E SUPERVISOR EDITORIAL

Ana Mara Ferreira Lima

e-mail:anamarafl@yahoo.com.br

\section{INTEGRANTES DA COMISSÃO CIENTÍFICA}

- PRESIDENTE

Ana Mara Ferreira Lima

\section{INTEGRANTES DA BANCA AVALIADORA}

\author{
Ana Paula da Silva \\ Djeanny Fortes Escorcio \\ Drayton Cavalcante Melo \\ Janara Cristina de Oliveira Soares \\ Kenia Mendes Rodrigues Castro \\ Rogério Moreira Junior \\ Sharlanderson da Costa Silva \\ Tasia Peixoto de Andrade Ferreira
}




\section{ESTRUTURA ADMINISTRATIVA}

- Diretora Geral

Maria do Carmo Amaral Brito

- Diretor Administrativo

Atila de Melo Lira

- Diretora de Ensino

Ivonalda Brito de Almeida Morais

- Coordenadora de Ensino

Maria das Graças de Melo Lira

- Coordenador de Apoio aos Cursos

José de Ribamar da Silva

- Coordenador do Curso de Bacharelado em Fisioterapia Gabriel Mauriz de Moura Rocha

- Secretária Acadêmica

Dariely de Carvalho Monte Amaral

- Coordenador Financeiro

José Gomes do Amaral Neto

- Coordenadora de Recursos Humanos

Najara Francélia de Brito Barbosa

- Coordenação Geral do NICEP

Sílvia Piva Rosal Morais

- Coordenação da Clínica escola - CHRISFAPI

Flávia Samara Freitas de Andrad 
ANAIS DA VIII JORNADA DE FISIOTERAPIA DA CHRISFAPI, 2019;02-38

\section{PALESTRAS E PALESTRANTES}

\begin{tabular}{|c|}
\hline $\begin{array}{c}\text { Raciocinio Clinico e conceito Maitland - Marcello de Alencar Silva } \\
\text { Treinamento Muscular Respiratório na prevenção de eventos cardiovasculares em } \\
\text { pacientes hemodialíticos - Nataly Gurgel Campos }\end{array}$ \\
$\begin{array}{c}\text { Abordagem Fisioterapêutica do tecido neural periférico - Francisco Elezier Xavier } \\
\text { Magalhães }\end{array}$ \\
\hline Atuação Fisioteraêutica no pós operatório de cirurgia cardiaca- \\
Francisco Maurilio da Silva Carrias \\
\hline Aneurisma Cerebral: Repercussões na Fisioterapia - Cáio César \\
Meneses de Sousa \\
\hline Neuromodulação: Evidências e perspectivas - Adriana Cavalcante \\
\hline Fisioterapia nas disfunções temporomandibulares - Júlia Moita Ferreira \\
\hline Distrofia Muscular de Duchenne - Eduarda Luna \\
\hline Efeito da fotobiomodulação em modelos experimentais de inflamação - Marcelo \\
de Carvalho Filgueiras
\end{tabular}

\section{MINICURSOS}

\begin{tabular}{|c|}
\hline $\begin{array}{c}\text { Atuação da Fisioterapia no pós operatório ao retorno ao esporte - Kelson Nonato } \\
\text { Gomes da Silva }\end{array}$ \\
\hline BLS: Suporte Básico de Vida - Djeanny Fortes Escórcio \\
\hline A importancia da ergonomia e do fisioterapeuta na escola - Carina Silva Moura \\
\hline Treinamento Muscular Respiratório - Jnataly Gurgel Campos \\
\hline Mobilização Neuromeningea - Francisco Elezier Xavier Magalhães \\
\hline Radiofrequência x Estrias: Avaliação e tratamento - Sayanne Kelly do Monte Silva
\end{tabular}




\section{SUMÁRIO}

ORGANIZADORES DO EVENTO

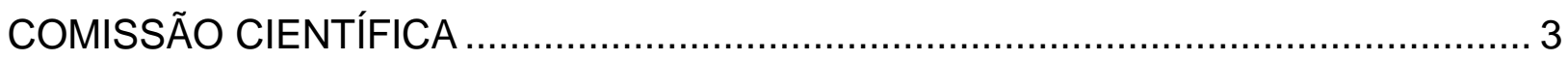

INTEGRANTES DA COMISSÃO CIENTÍFICA ....................................................... 3

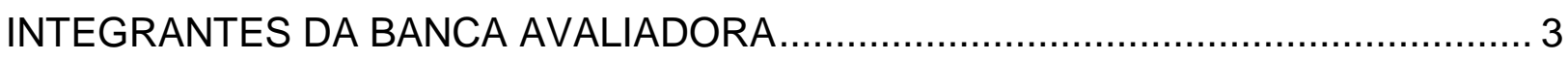

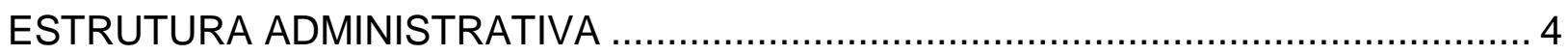

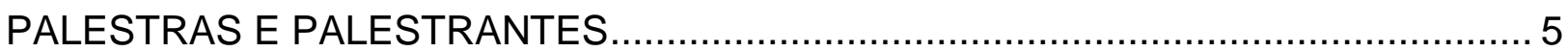

ANÁLISE DOS EFEITOS DA VENTILAÇÃO NÃO-INVASIVA (VNI) NO PÓSOPERATÓRIO DE CIRURGIAS CARDÍACAS: revisão de literatura ............................ 7

A EFICÁCIA DA TERAPIA ESPELHO NO TRATAMENTO DE MEMBROS PARÉTICOS EM PACIENTES PÓS - ACIDENTE VASCULAR ENCEFÁLICO: revisão integrativa.

EFICÁCIA DA ACUPUNTURA NA REDUÇÃO DOS SINTOMAS EM PACIENTES COM DISFUNÇÃO TEMPOROMANDIBULAR (DTM): revisão integrativa

APLICABILIDADE DO CONCEITO MULLIGAN EM ENTORSES DE TORNOZELOS E SEUS BENEFÍCIOS FISIOLÓGICOS E BIOMECÂNICOS ...................................... 14 EFICÁCIA DO TRATAMENTO FISIOTERAPÊUTICO NO EQUILIBRIO E MARCHA DE INDIVIDUOS COM DOENÇA DE PARKINSON: revisão de literatura ASSISTÊNCIA FISIOTERAPÊUTICA NO TRABALHO DE PARTO E PARTO: revisão integrativa.

UTILIZAÇÃO DA TERAPIA MANUAL NO TRATAMENTO DA LOMBALGIA PROVOCADA PELA COMPRESSÃO DO MÚSCULO PIRIFORME.

A EFICÁCIA DO TREINAMENTO DE FORÇA MUSCULAR REALIZADO COM OCLUSÃO VASCULAR

A INTERVENÇÃO DA FISIOTERAPIA NA QUALIDADE DE VIDA DE MULHERES MASTECTOMIZADAS: revisão integrativa 25

O USO DA ACUPUNTURA NO TRATAMENTO DA DEPRESSÃO: revisão integrativa

O USO DA ELETROESTIMULAÇÃO TRANSCUTÂNEA DO NERVO TIBIAL NO TRATAMENTO DE PACIENTES COM BEXIGA HIPERATIVA: revisão integrativa..... 29 ANÁLISE DA QUALIDADE DE VIDA E DA CAPACIDADE FUNCIONAL DE

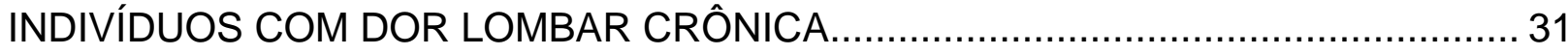
TUBERCULOSE: uma abordagem geral dos principais aspectos ............................. 33 A UTILIZAÇÃO DA VENTOSATERAPIA NA REDUÇÃO DO FIBRO EDEMA GELÓIDE - TEORIA CIRCULATÓRIA 36 A ATUAÇÃO DA FISIOTERAPIA EM MULHERES COM DISMENORREIA PRIMÁRIA: revisão integrativa 
ANAIS DA VIII JORNADA DE FISIOTERAPIA DA CHRISFAPI, 2019;02-38

\section{ANÁLISE DOS EFEITOS DA VENTILAÇÃO NÃO-INVASIVA (VNI) NO PÓS- OPERATÓRIO DE CIRURGIAS CARDÍACAS: revisão de literatura}

Andrei lago Gonçalves Viana Soares Feitosa ${ }^{1}$, João Paulo Castro Soares ${ }^{\star 1}$, Carlos Eduardo Nunes Vieira ${ }^{1}$, Paulo Roberto Milanez Oliveira Junior ${ }^{2}$

${ }^{1}$ Graduandos do Curso de Fisioterapia pela Faculdade Maurício de Nassau,

${ }^{2}$ Fisioterapeuta e orientador do artigo

\section{RESUMO}

INTRODUÇÃO: A cirurgia cardíaca é considerada um procedimento que possibilita a remissão dos sintomas, contribuindo para o aumento da sobrevida e melhora da qualidade de vida dos indivíduos cardiopatas. OBJETIVO: Identificar por meio de uma revisão de literatura os efeitos da VNI no PO imediato de cirurgias cardíacas. MÉTODOS: Revisão de literatura de artigos nas bases de dados LILACS, SciELO, MedLine/PubMed e PEDro, utilizando os descritores "sternotomy", "physiotherapy", "noninvasive ventilation" e "thoracic surgery" com o operador boleano "and", publicados entre 2009 a 2019. RESULTADOS: Foram encontrados 20 estudos, 14 foram excluídos, por não possuírem o delineamento metodológico estipulado no presente estudo. Foram incluídos seis ensaios clínicos que contemplaram os critérios estabelecidos para o desfecho pretendido. As informações sobre os estudos inseridos encontram-se sintetizadas no quadro 1. Três estudos utilizaram grupo controle para comparação dos resultados. O tamanho amostral variou entre 30 a 284 voluntários no pós-operatório imediato de cirurgias cardíacas, com média de idade variando entre 18 e 80 anos, predominantemente do sexo masculino (61,77\%), submetidos à diferentes modalidades de VNI. As intervenções cirúrgicas fazem parte da terapêutica atual das cardiopatias (PREISIG et al., 2014). Por conta das complicações a VNI promove a diminuição do trabalho respiratório, do índice de dispneia e aumento do volume residual, prevenindo assim a presença de atelectasias, além de favorecer o recrutamento alveolar e a $\mathrm{PaO}_{2}$ (TOME et al., 2018). Franco et al. (2011) e Daniel et al. (2014) afirmam que o uso da VNI em complicações respiratórias (pneumonias e atelectasias) no PO, colaboram com a redução do tempo de internação e de UTI. Tal fato deve a redução do trabalho respiratório e o aumento da complacência do sistema respiratório, por reverter os quadros de complicações do pulmão, não dependendo do esforço do paciente para gerar inspirações profundas, sendo 
ANAIS DA VIII JORNADA DE FISIOTERAPIA DA CHRISFAPI, 2019;02-38

assim uma vantagem em relação a outros métodos, principalmente em PO imediato, no qual o paciente é pouco cooperativo ou incapaz de realizar inspiração máxima devido à dor, promovendo aumento dos valores de volumes e capacidades pulmonares (FRANCO et al., 2011; DANIEL et al., 2014). Já Tome et al. (2018) afirmam que não acreditam que a cirurgia torácica aberta possa influenciar a ausência de relação entre ventilação e VVS porque observamos que, à medida que a ventilação dos dois pulmões foi restaurada (com pleura aberta), a relação entre os parâmetros respiratório e cardíaco foi restabelecida. CONCLUSÃO: O uso e administração da VNI foi eficaz e seguro para os pacientes no PO de cirurgia cardíaca. Vale ressaltar que isso se deve a boa adaptação e respostas positivas do paciente as diferentes modalidades, colaborando com a redução do tempo de internação e de UTI.

Palavras-Chave: Esternotomia. Fisioterapia. Ventilação não-invasiva. Cirurgia torácica

\section{REFERÊNCIAS}

OliveiRA, M. A. B.; BOTELHO, P. H. H.; BRANDI, A. C.; SANTOS, C. A.; SOARES, M. J. F.; ZAIANTCHICK, M. et al. Importância da troponina I no diagnóstico do infarto do miocárdio no pós-operatório de cirurgia de revascularização. Rev Bras Cir Cardiovasc., v. 24, n. 1, p. 11-14, 2009.

MAZULLO FILHO, J. B. R.; BONFIM, V. J. G.; AQUIM, E. E. Ventilação mecânica não invasiva no pós-operatório imediato de cirurgia cardíaca. Rev. bras. ter. intensiva, v. 22, n. 4, p. 363-368, 2010.

LAIZO, A.; DELGADO, F. E. F.; ROCHA, G. M. Complicações que aumentam o tempo de permanência na unidade de terapia intensiva na cirurgia cardíaca. Rev Bras Cir Cardiovasc., v. 25, n. 2, p. 166-171, 2010.

FRANCO, A. M. et al. Avaliação da ventilação não-invasiva com dois níveis de pressão positiva nas vias aéreas após cirurgia cardíaca. Rev Bras Cir Cardiovasc, São José do Rio Preto, v. 26, n. 4, p. 582-590, 2011.

]

CAVALLI, F.; NOHAMA, P. Novo dispositivo EPAP subaquático no pós-operatório de cirurgia de revascularização do miocárdio. Fisioter. mov., v. 26, n. 1, p. 37-45, 2013.

PREISIG, A. et al. Ventilação não Invasiva após Cirurgia Cardiovascular: um Ensaio Clínico Randomizado. Rev Bras Cardiol., v. 27, n. 1, p. 43-52, 2014.

SUEHIRO, K.; OKUTANI, R. Stroke volume variation as a predictor of fluid responsiveness in patients undergoing one-lung ventilation. J Cardiothorac Vasc Anesth., v. 24, p. 772-775, 2010.

SILVEIRA, A. P. C. et al. Comparação do uso da pressão positiva com a fisioterapia convencional e incentivadores respiratórios após cirurgia cardíaca: revisão de literatura. 
ANAIS DA VIII JORNADA DE FISIOTERAPIA DA CHRISFAPI, 2019;02-38

Medicina (Ribeirão Preto), v. 44, n. 4, p. 338-346, 2011.

TOME, M. L. et al. Behavior of stroke volume variation in hemodynamic stable patients during thoracic surgery with one-lung ventilation periods. Rev. Bras. Anestesiol., v. 68, n. 3, p. 225230, 2018.

ALCÂNTARA, E. C.; NAVES-SANTOS, V. Estudo das complicações pulmonares e do suporte ventilatório não invasivo no pós-operatório de cirurgia cardíaca. Rev Med Minas Gerais, v. 19, n. 1, p. 5-12, 2009.

DE BACKER, D. et al. Influence of respiratory rate on stroke volume variation in mechanically ventilated patients. Anesthesiology, v. 110, p. 1092-1097, 2009.

TREPTE, C. et al. Prediction of volume-responsiveness during one-lung ventilation: a comparison of static, volumetric, and dynamic parameters of cardiac preload. $\mathbf{J}$ Cardiothorac Vasc Anesth., v. 27, p. 1094-1100, 2013.

DANIEL, C. R. et al. Respiratory muscle strength no influence the need for noninvasive ventilation after heart surgery. Fisioter. Pesqui., São Paulo, v. 21, n. 1, p. 60-66, 2014. 
ANAIS DA VIII JORNADA DE FISIOTERAPIA DA CHRISFAPI, 2019;02-38

\section{A EFICÁCIA DA TERAPIA ESPELHO NO TRATAMENTO DE MEMBROS PARÉTICOS EM PACIENTES PÓS - ACIDENTE VASCULAR ENCEFÁLICO: revisão integrativa}

Carolyne Carvalho Caxias ${ }^{1}$; Ana Paula da Silva Carvalho²; Luís Mário Rezende Júnior²

1 Graduanda do Curso de Fisioterapia pela Cristo Faculdade do Piauí - CHRISFAPI

2 Docente do Curso de Fisioterapia pela Cristo Faculdade do Piauí - CHRISFAPI

\section{RESUMO}

INTRODUÇÃO: O sinal mais comum do AVE na fase adulta é a hemiparesia da face, braço e/ou perna. A Terapia do Espelho tem sido referenciada como um método de tratamento inovador na tentativa de minimizar as alterações sensoriais e motoras bem como acelerar o processo de recuperação funcional de membros paréticos. OBJETIVO: Analisar, por meio de uma revisão integrativa, os efeitos da terapia espelho no tratamento de membros paréticos de indivíduos que sofreram acidente vascular encefálico. METODOLOGIA: Para a realização deste estudo foram realizadas buscas na base de dados eletrônicas Biblioteca Virtual em Saúde (BVS), Literatura Latino-Americana e do Caribe em Ciências da Saúde (LILACS), Scientific Electronic Library Online (SciELO), na base de dados de Evidências em Fisioterapia (PEDro) e Medical Literature Analysis and Retrieval System Online (MEDLINE). Para a pesquisa foram utilizados de forma associada os descritores "fisioterapia", "AVE", e "terapia espelho". Os critérios utilizados para seleção dos artigos foram trabalhos disponibilizados de forma completa e gratuita na língua portuguesa, inglesa ou espanhola, publicados entre 2013 e 2019, que abordem a TE em pacientes sequelados por AVE. RESULTADOS E DISCUSSÃO: Após a leitura criteriosa dos 20 artigos encontrados apenas 06 atenderam aos critérios de inclusão. Sendo 02 artigos na língua inglesa e 04 na língua portuguesa. Os resultados obtidos mostraram aumento da função e controle motor do membro parético, melhora da funcionalidade, diminuição da espasticidade, aumento da velocidade na execução das atividades de vida diária, entretanto, alguns indivíduos não apresentaram resultados estatísticos significativos. CONCLUSÃO: A terapia do espelho foi eficaz para a diminuição do quadro álgico, melhora da motricidade fina e grossa dos membros paréticos possibilitando ganhos funcionais, bem como diminuição do tempo na realização de atividades. No entanto, faz-se necessário novos estudos acerca da temática estudada para elucidar possíveis falhas nos procedimentos aplicados. 
Palavras-chave: Fisioterapia. AVE. Terapia Espelho.

\section{REFERÊNCIAS}

LIMA, E. O.; ANDRADE, T. M.; MELO, G. A et al. Analysis of motor activity in hemiplegic submitted to therapy mirror: case reports. Revista Neurociências. V. 23, n. 3, p. 436-442, 2015.

MEDEIROS, C. S. P.; FERNANDES, S. G. G.; LOPES, J. M. et al. Effects of mirror therapy through functional activities and motor standards in motor function of the upper limb after stroke. Revista Fisioterapia e Pesquisa. V. 21, n. 3, p. 264-270, 2014.

MELO, L. P.; BEZERRA, V. T.; COSTA, V. S. et al. Efeitos da terapia espelho na reabilitação do membro superior pós-acidente vascular cerebral. Revista Saúde (Santa Maria). Santa Maria, v. 41, n. 1, p. 157-164, jan./jul. 2015.

PAULINO, R. H.; PASTOR, F. A. C. Feedback visual com espelho em membro inferior parético após acidente vascular encefálico: Estudo de Casos. Revista Inspirar Movimento \& Saúde. V. 6, n. 5, out./nov./dez. 2014.

SILVEIRA, J. C. C.; COSTA, V. S.; CLEMENTINO, T. C. A. et al. Função motora melhora em pacientes pós-acidente vascular cerebral submetidos à terapia espelho. Revista de Terapia Ocupacional da Universidade de São Paulo. V. 28, n. 3, p. 333-9; set./dez. 2017. 


\title{
EFICÁCIA DA ACUPUNTURA NA REDUÇÃO DOS SINTOMAS EM PACIENTES COM DISFUNÇÃO TEMPOROMANDIBULAR (DTM): revisão integrativa
}

\author{
Larissa Kelly Carvalho da Silva ${ }^{1 *}$, Sanny Maria Pereira da Silva ${ }^{1}$, Shirley Pontes da Silva ${ }^{1}$, \\ Daiany Sousa Monteiro² \\ 1 Instituição: Cristo Faculdade do Piauí - CHRISFAPI. \\ 2 Docente da Cristo Faculdade do Piauí - CHRISFAPI.
}

\section{RESUMO}

INTRODUÇÃO: A disfunção temporomandibular (DTM) é um termo que abrange vários problemas clínicos, que envolvem a musculatura mastigatória. As manifestações decorrentes da DTM ocorrem em torno de $33,3 \%$ das pessoas, podendo inclusive comprometer a qualidade de vida das pessoas acometida. Com isso a acupuntura é uma das técnicas utilizadas no tratamento das alterações ocasionadas. Baseia-se na estimulação de pontos anatômicos específicos do corpo denominados acupontos. Esta técnica age estimulando a liberação de opióides pelo organismo, agindo no controle da dor. OBJETIVO: Esse estudo tem por objetivo identificar a eficácia da acupuntura no tratamento dos sintomas ocasionados por disfunções temporomandibulares. METODOLOGIA: O estudo se trata de uma revisão integrativa que aborda a atuação da acupuntura na redução dos sintomas em pacientes com DTM, através da base de dados: Scielo, PEDro e MedLine. Sendo os critérios de inclusão: Artigos científicos publicados entre 2014 e 2019, escritos em língua portuguesa e inglesa. RESULTADOS: Com a busca, 7 estudos foram encontrados. Após as análises dos textos, foram selecionados 4 trabalhos. Os quais foram inclusos de acordo com os critérios de inclusão. Nestes foram observados que realizaram avaliações antes do tratamento, seguida das intervenções no qual aplicaram a técnica nos pontos correspondentes ao reequilíbrio emocional. Com a reavaliação dos resultados dos tratamentos foi constatado que ambos os tratamentos estudados apontaram a redução da intensidade da dor e aumento da abertura bucal, melhorando os movimentos mandibulares. CONCLUSÃO: Nesse trabalho pode-se identificar que o uso da acupuntura foi de importante significância na resolução dos sinais e sintomas em pacientes desta disfunção. Portanto a sua utilização poderia contribuir para ampliar o atendimento a pacientes com DTM.

\footnotetext{
${ }^{1}$ Graduanda do III período do Curso de Fisioterapia da Cristo Faculdade do Piauí - CHRISFAPI

${ }^{2}$ Orientadora e Docente do Curso de Fisioterapia da Cristo Faculdade do Piauí - CHRISFAPI
} 
ANAIS DA VIII JORNADA DE FISIOTERAPIA DA CHRISFAPI, 2019;02-38

Palavras-chave: Dor orofacial. Acupuntura. Articulação temporomandibular.

\section{REFERÊNCIAS}

CAMARGO, B. A. B.; GRILLO, C. M.; SOUSA, M. L. R. Redução da dor da disfunção temporomandibular com acupuntura: estudo descritivo longitudinal preliminar. Revista Dor. Vol. 15, n. 03, p. 159-162, 2014.

DANTAS, A. M. X.; SANTOS, E. J. L.; VILELA, R. M. et al. Perfil epidemiológico de pacientes atendidos em um serviço de controle da dor orofacial. Revista de Odontologia na UNESP. Vol. 44, n. 6, p. 313-319, 2015.

GRILLO, C. M.; CANALES, G. D. L. T.; WADA, R. S. et al. Could acupunture beuseful in the treatment of temporo mandibular dysfunction? Journal of Acupuncture and Meridian Studies. Vol. 08, n. 04, p. 192-199, 2015.

MENDES, K. S.; SILVEIRA, R. C. C. P.; GALVÃO, C. M. Revisão Integrativa: Método de pesquisa para a incorporação de evidênciasna saúde e na enfermagem. Texto \& Contexto Enfermagem.Vol. 17, n. 4, p. 758-764, 2008.

SOUSA, M. L.; MASHUDA, C. S.; SATO, J. E.; SIQUEIRA, J. T. T. Efeito da acupuntura em adultos com disfunção temporomandibular. Revista Dor. Vol. 15, n. 02, p. 87-90, 2014. ZOTELLI, V. L.; GRILLO, C. M.; GIL, M. L. et al. Acupunture effectonpain, mouth opening limitation and on the energy meridians in patients with temporo mandibular dysfunction: a randomized controlled trial. Journal of Acupuncture and Meridian Studies. Vol. 10, n. 05, p. 351-359, 2017. 


\title{
APLICABILIDADE DO CONCEITO MULLIGAN EM ENTORSES DE TORNOZELOS E SEUS BENEFÍCIOS FISIOLÓGICOS E BIOMECÂNICOS
}

\author{
Wellen Yara Silva Santos ${ }^{1 *}$, Míriam Alves Silva ${ }^{1}$, Renan do Nascimento Silva ${ }^{1}$, Kênia Mendes \\ Rodrigues Castro ${ }^{2}$ \\ 1 Instituição: Cristo Faculdade do Piauí - CHRISFAPI, \\ 2 Docente da Cristo Faculdade do Piauí - CHRISFAPI.
}

\section{RESUMO}

INTRODUÇÃO: A lesão por entorse de tornozelo são caracterizadas por alongamento das fibras dos ligamentos da articulação do tornozelo. São lesões comuns no esporte, com probabilidade de ocorrência maior em indivíduos entre 21 a 30 anos. As entorses são classificadas em grau 1 (estiramento dos ligamentos) grau 2 (ruptura parcial dos ligamentos) grau 3 (ruptura total dos ligamentos). O Conceito Mulligan combina mobilização articular passiva com ativas a fim de restaurar o padrão fisiológico da articulação acometida sem provocar dor, neste caso as mobilizações em movimento (MWM) atuam restaurando os desequilíbrios provocados pela lesão. OBJETIVO: Verificar através da literatura quais os possíveis benefícios que são adquiridos ao introduzir a técnica MWM em entorses de tornozelo. METODOLOGIA: Trata-se de uma revisão integrativa conduzida por busca em bancos de dados incluindo SCIELO (Scientific Electronic Library Online), PUBMED (Publisher Medline), LILACS (Literatura Latino-Americana em Ciências da Saúde), MEDLINE (Medical Literature Analysis and Retrieval System Online) e PeDRO (Base de Dados em Evidências em Fisiorerapia), após a análise foram selecionados 7 estudos. Como critérios de inclusão foram utilizados artigos originais que abordassem o tema com delineamento experimental realizados em humanos, datados de 2010 a 2019 e critérios de exclusão foram artigos que não fosse gratuito ou que não estivesse completo na íntegra, pagos e que não se enquadrava no tema proposto. RESULTADOS: Com a análise dos estudos foi possível verificar benefícios fisiológicos e biomecânicos associados à intervenção das técnicas MWM, com destaque para redução do quadro álgico, ganho de amplitude de movimento para dorsiflexão e funcionalidade que se evidenciaram de forma significativa em períodos tardios. CONCLUSÃO: O estudo em questão demostrou ser de grande valia para a compreensão dos efeitos positivos da utilização do método Mulligan nos entorses de tornozelo ocasionados por inversão do pé. 
ANAIS DA VIII JORNADA DE FISIOTERAPIA DA CHRISFAPI, 2019;02-38

Palavras-chaves: Fisioterapia. Entorses de Tornozelo. Terapia manual.

\section{REFERÊNCIAS}

BECHE, Aline Oliveira. Efeitos imediatos da manipulação e mobilização em jovens com instabilidade crónica de tornozelo. Dissertação submetida à Escola Superior de Saúde para obtenção do grau de Mestre em Fisioterapia. Porto, nov 2018.

BRUMITT, Jason. Casos clínicos em fisioterapia esportiva. Porto Alegre: AMGH, 2017. CALAÇA, Frederico Igor Ribeiro. Influência da técnica mobilização com movimento e tape de Mulligan em indivíduos com instabilidade de tornozelo: Ensaio clínico randomizado e cego. Brasília, p.35, 2017.

CANEIRO, Miguel Martins; et al. Efeitos da mobilização com movimento da tíbioperonial inferior e da sua combinação com o astrágalo, em entorses crónicas do tornozelo.

Dissertação submetida à Escola Superior de Saúde para obtenção do grau de Mestre em Fisioterapia, Porto: 2017.

DUTTON, Mark. Fisioterapia Ortopédica: Exame, avaliação e intervenção. 2.ed. Porto Alegre: Artmed, 2010.

HUDSONA, Robinetta; et al.Novel treatment of lateral ankle sprains using the Mulligan concept: an exploratory case series analysis. Journal of Manual \& Manipulative Therapy, 2017. Vol . 25, No . 5, 251-259.

JUNIOR, Adroaldo José Casa; MEDEIROS, Raissa Netto. Efetividade do conceito mulligam na entorse de tornozelo em inversão. Revista brasileira de trabalhos acadêmicosUniverso. Goiânia. Volume1, nº 1, 2016.

JUNIOR, Adroaldo José Casa; REZENDE, Lauanny Pires; CASA, Nara Lígia Leão. Efeitos do Conceito Mulligan na dor e funcionalidade na síndrome do ombro doloroso. Revista Movimenta. ISSN:1984-4298 2018; 11(2):147-154.

JÚNIOR, Newton da Silva Pereira; ALMEIDA Rogério Moreira de. Manual de recursos terapêuticos manuais. João Pessoa: Editora da UFPB, 2016.

MACMAHON, Patrick J. CURRENT: Diagnóstico e tratamento em medicina do esporte. Porto Alegre: Artmed, 2011.

PAÇO, Maria. Efeitos imediatos da técnica de mobilização com movimento aplicada na articulação tibio-peroneal inferior na amplitude de dorsiflexão em indivíduos com história de entorse do tornozelo. Relatório do Projecto de Investigação apresentado para obtenção do grau de Mestre em Fisioterapia, Satúbal, 2011.

SANTOS, Joana Sousa; AMARAL Luísa. Kinesio taping vs. Técnica de mulligan na reabilitação da instabilidade crônica da articulação tibiotarsica em atletas de andebol: estudo randomizado. Porto, 2017.

SIMON Robert R.; SHERMAN Scott C. Emergências Ortopédicas. 6.ed. Porto Alegre: 
ANAIS DA VIII JORNADA DE FISIOTERAPIA DA CHRISFAPI, 2019;02-38

Artmed, 2013.

SIVAKUMAR, C.; et al. Effectiveness strengthening exercises and Mulligan's mobilization in patients with unilateral ankle sprain. Int. J. Res. Pharm. Sci., 8 (4), 555-559, 2017. 
ANAIS DA VIII JORNADA DE FISIOTERAPIA DA CHRISFAPI, 2019;02-38

\title{
EFICÁCIA DO TRATAMENTO FISIOTERAPÊUTICO NO EQUILIBRIO E MARCHA DE INDIVIDUOS COM DOENÇA DE PARKINSON: revisão de literatura
}

\author{
Élita Sampaio Emiliano1, Geysa Brandão Dos Santos', Andreza Judith Magalhães do \\ Amaral $^{1}$, Yago Felipe Rodrigues Pinto ${ }^{1}$, Ana Paula da Silva Carvalho². \\ 1 Instituição: Cristo Faculdade do Piauí - CHRISFAPI, \\ 2 Docente da Cristo Faculdade do Piauí - CHRISFAPI.
}

\section{RESUMO}

INTRODUÇÃO: O Parkinson é uma doença neurodegenerativa, que causa desordem crônica, progressiva e degenerativa no sistema nervoso central e sua incidência é maior em homens, com idade média de 60 anos. Os pacientes com a doença de Parkinson (DP), apresentam clinicamente, bradicinesia, tremor em repouso, rigidez articular, instabilidade postural e comprometimento da capacidade de executar tarefas. A fisioterapia promoverá benefícios, pois englobará exercícios terapêuticos de alongamento, fortalecimento muscular, transferências, relaxamento, marcha, equilíbrio, mobilidade. O objetivo desse estudo é analisar por meio da literatura as formas de tratamento fisioterapêutico nesta patologia. METODOLOGIA: Realizou-se no período de abril a maio de 2019 uma revisão sistemática das publicações indexadas nas bases de dados Scielo nos últimos 10 anos, nos idiomas português e inglês. Os critérios de inclusão foram os estudos que abordassem o tratamento fisioterapêutico no equilíbrio e marcha e artigos de revistas que apresentassem qualis até B3. Nos critérios de exclusão, foram os artigos que não apresentavam clareza quanto ao método utilizado e resultados, e artigos de revistas que apresentassem qualis inferior a B3. RESULTADOS: Neste estudo de revisão de literatura foram encontrados 12 estudos no total. Destes, 4 artigos foram excluídos por se tratarem de estudos antigos. Entre 8 restantes, 3 foram excluídos por terem temas que não abordavam interesse fisioterapêutico, restando assim, 5 artigos encontrados na base de dados Scielo. CONCLUSÃO: Diante do que foi visto, pode-se perceber que os tratamentos fisioterapêuticos obtiveram uma melhora significativa no equilíbrio e marcha de sujeitos com DP. Sendo importante buscar novas pesquisas com técnicas desenvolvidas pela fisioterapia para promover a melhora da qualidade de vida dos mesmos.

Palavras-chaves: Doença de Parkinson. Equilíbrio Postural. Fisioterapia. 


\section{REFERÊNCIAS}

BRAZ, N. F. T. et al., Eficácia do Nintendo Wii em desfechos funcionais e de saúde de indivíduos com doença de Parkinson: uma revisão sistemática. Revista Fisioterapia e Pesquisa, v. 25, n. 1, p. 100-106, 2018.

GONDIM, I. T. G. O., LINS, C. C. S. A., CORIOLANO, M. G. W. S. Exercícios terapêuticos domiciliares na doença de Parkinson: uma revisão integrativa. Revista Brasileira de Geriatria Gerontologia, v. 19, n. 2, p. 349-364, Rio de Janeiro, 2016.

MONTEIRO, D. et al., Prática mental após fisioterapia mantêm mobilidade funcional de pessoas com doença de Parkinson. Revista Fisioterapia e Pesquisa, v. 25, n. 1, p. 65-73, 2018.

MONTEIRO, E. P. et al., Aspectos biomecânicos da locomoção de pessoas com a doença de Parkinson: revisão narrativa. Revista Brasileira de Ciência e Esporte, v. 39, n. 4, p. 450-457, 2017. 
ANAIS DA VIII JORNADA DE FISIOTERAPIA DA CHRISFAPI, 2019;02-38

ASSISTÊNCIA FISIOTERAPÊUTICA NO TRABALHO DE PARTO E PARTO: revisão integrativa

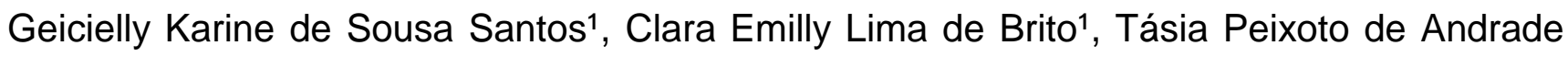
Ferreira²

1 Instituição: Cristo Faculdade do Piauí - CHRISFAPI,

2 Docente da Cristo Faculdade do Piauí - CHRISFAPI.

\section{RESUMO}

INTRODUÇÃO: A assistência obstétrica demonstra grande importância, a qual atenda seus aspectos de: acolhimento, acesso, resolutividade e qualidade. Sendo fundamental a presença do fisioterapeuta neste momento, pois tem como ocupação monitorar e avaliar as alterações físicas focando na manutenção do bem-estar da parturiente e do bebê, mediante seus inúmeros recursos terapêuticos. OBJETIVO: Revisar artigos que avaliam os benefícios da fisioterapia durante o trabalho de parto. METODOLOGIA: Os descritores utilizados foram fisioterapia, trabalho de parto e assistência ao parto. Teve-se como $\mathrm{Cl}$ artigos encontrados de forma integral e gratuito, com os mesmos descritores, nas línguas inglês e português. Quanto aos CE: foram descartados artigos publicados antes de 2013, também foram excluídos artigos de revisão e os artigos duplicados nas bases de dados. RESULTADOS: Diante do verificado, fez-se possível destacar intervenções de redução da dor no trabalho de parto e até mesmo no momento do parto. CONCLUSÃO: Pode-se concluir que a assistência fisioterapêutica ao trabalho de parto e parto é um método eficaz para a melhoria da qualidade do parto, redução da dor, aumento da segurança e conforto da parturiente.

Palavras-chave: Fisioterapia. Trabalho de Parto. Assistência ao Parto. 


\section{REFERÊNCIAS}

ABREU, Nathalia et al. Atenção fisioterapêutica no trabalho de parto e parto. Revista Interdisciplinar de Estudos Experimentais-Animais e Humanos Interdisciplinary Journal of Experimental Studies, v. 5, 2013.

AKBARZADEH, M. et al. Comparison of the effects of maternal supportive care and acupressure (BL32 acupoint) on pregnant women's pain intensity and delivery outcome. Journal of pregnancy, v. 2014, 2014.

BARBIERI, M. et al. Banho quente de aspersão, exercícios perineais com bola suíça e dor no trabalho de parto. Acta Paulista de Enfermagem, v. 26, n. 5, 2013. 


\section{UTILIZAÇÃO DA TERAPIA MANUAL NO TRATAMENTO DA LOMBALGIA PROVOCADA PELA COMPRESSÃO DO MÚSCULO PIRIFORME}

${ }^{1}$ Karen de Carvalho Brito*, Brenda Maria de Sousa Andrade'1, Káren Andressa Mendes da Silva ${ }^{1}$, Maria Thalya da Silva Lopes ${ }^{1}$, Brenda Kellen de Sousa Martins ${ }^{1},{ }^{2}$ lara Tamires Ibiapina 1 Discentes do Curso de Fisioterapia da Cristo Faculdade do Piauí - CHRISFAPI, Piripiri- PI 2Preceptora de estágio na clínica escola Chrisfapi

\section{RESUMO}

INTRODUÇÃO: A dor lombar define-se por uma fadiga muscular, rigidez muscular ou quadro de desconforto no terço inferior da coluna vertebral, podendo ou não ter irradiação para os membros inferiores com intensidade e duração variável. A terapia manual é afamada como um recurso no sobreaviso das dores, cansaço físico e mental, se trata de um recurso manual não invasivo que melhora a circulação sanguínea, linfática, o metabolismo e alivia tensões. OBJETIVO: Este estudo teve o proposto objetivo de demostrar a importância da utilização da terapia manual no tratamento da dor provocada pela compressão no músculo piriforme. METODOLOGIA: O presente artigo de revisão integrativa foi realizado uma consulta em base de dados como: Lilacs, Medline, Pubmed, Scielo, utilizando como descritores "Massoterapia"; "Lombalgia"; "Fisioterapia". Como critério de inclusão foi selecionado artigos de relato de caso envolvendo a temática, artigos que se enquadrava nos períodos de 2008 a 2019. Como critérios de exclusão artigos que tratavam de outra abordagem terapêutica, ou que não se enquadravam dentro do período proposto, sendo encontrado um total de 12 artigos. RESULTADOS E DISCUSSÃO: Nos resultados encontrados foi possível perceber, que a técnica de massoterapia, contribuiu para o tratamento da lombalgia, tendo assim a melhora do quadro. CONCLUSÃO: Após os estudos comparados conclui-se que dos quatro artigos, três relacionou-se com a temática mostrando que teve um real benefícios da terapia manual no tratamento da lombalgia.

Palavra-chave: Massoterapia. Lombalgia. Fisioterapia. 


\section{REFERÊNCIAS}

ABREU, M.F. et al. Síndrome do piriforme, lombalgia ou lombociatalgia. Existe diferença? Revista Estação Científica, Juiz de fora, n. 14, p. 1-15, jul, 2015. Disponível em: $<$ http://portal.estacio.br/docs\%5Crevista_estacao_cientifica/05-14.pdf>. Acessado em: 15 de nov de 2017

ALBRECHT, Bruna Schwingel; DA LUZ GOULART, Cassia; WEIS, Luciana Cezimbra. Análise da melhora de dor em pacientes com lombalgia submetidos a procedimentos fisioterapêuticos. Revista Jovens Pesquisadores, Santa Cruz do Sul, v. 5, n. 3, p. 17-25, 2015.Disponível em:

<http://online.unisc.br/seer/index.php/jovenspesquisadores/article/view/5704>. Acessado em: 14 de nov de 2017

ALVES CP, Lima EA. Guimarães RB. Tratamento fisioterapêutico da lombalgia postural estudo de caso. Rev. Interfaces. 2014;2(6):1-4.

BRUM, Kazumi N.; ALONSO, Angélica C.; BRECH, Guilherme C. Tratamento de massagem e acupuntura em corredores recreacionais com síndrome do piriforme. Arquivo Ciência e Saúde, [S.L.] v. 16, n. 2, p. 62-66, abr-jun, 2009. Disponível em:

<http://files.marcosmartini.webnode.com.br/2000007508c4e48d484/Acupuntura\%20Sindro me\%20do\%20Piriforme.pdf >. Acessado em: 16 de nov de 2017. 
ANAIS DA VIII JORNADA DE FISIOTERAPIA DA CHRISFAPI, 2019;02-38

\section{A EFICÁCIA DO TREINAMENTO DE FORÇA MUSCULAR REALIZADO COM OCLUSÃO VASCULAR}

Rielle Nascimento Mesquita Silva1; Caio César de Sampaio Caetano Lima1; Ingrid Raissa Sousa Chaves"1; Erika Lages de Aguiar'; Ana Paula da Silva Carvalho²

${ }^{1}$ Discentes do Curso de Fisioterapia da Cristo Faculdade do Piauí - CHRISFAPI, Piripiri- PI 2Preceptora de estágio na clínica escola Chrisfapi

\section{RESUMO}

INTRODUÇÃO: O "Kaatsu training" se aplica através de uma força externa de compressão na proximidade dos membros, associada com esforços musculares de baixa intensidade e uma oclusão parcial vascular. Assim, possibilitando uma maior aglomeração de metabólitos produzidos durante o esforço, gerando uma adaptação fisiológica momentânea. Essa revisão visa verificar a efetividade do treinamento de força muscular por meio da oclusão vascular, além de identificar outros benefícios dessa técnica e analisar seus efeitos diante dos exercícios físicos. METODOLOGIA: O presente estudo trata-se de uma revisão bibliográfica. Os artigos selecionados sofreram critérios de inclusão, sendo eles artigos de 2014 a 2019 , Qualis A1 que estivessem disponíveis na língua portuguesa e estrangeira. RESULTADOS E DISCUSSÃO: Foram encontrados cerca de 62 artigos e selecionados para o presente estudo, 05 artigos. Sendo que nos estudos observou-se que a técnica contribui na melhora e tem impacto direto na funcionalidade desses indivíduos, podendo prevenir comprometimentos em suas qualidades de vida, além de melhorar significativamente sua força muscular. CONCLUSÃO: Com esse estudo, foi possível alcançar os objetivos, facilitando a melhor compreensão sobre o uso da oclusão venosa para ganho de resistência e força muscular. Pois com os resultados encontrados mostrou sua efetividade utilizando-se de pequenas cargas, além de proporcionar a melhora do quadro álgico durante as atividades.

Palavra-chaves: Resistência vascular. Fisioterapia. Oclusão terapêutica.

\section{REFERÊNCIAS}

BRYK, F. F. et al. Exercícios com oclusão vascular parcial em pacientes com osteoartrite do joelho: um estudo clínico aleatório. Knee Surg Sports Traumatol Arthrosc. v. 24, n. 5, p. 1580-1586, 2016. 
ANAIS DA VIII JORNADA DE FISIOTERAPIA DA CHRISFAPI, 2019;02-38

CAMARGO, G. et al. Treinamento físico com oclusão vascular: uma revisão sistematizada. Revista Científica Fagoc Saúde - Volume II - 2017. Disponível em:<http://revista.fagoc.br/index.php/saude/article/view/137>. Acesso em 02 Maio 2019.

Clarkson Matthew John, Conway Louise, Warmington Stuart Anthony. Blood fluxo curta restrição e função física em adultos mais velhos: um ensaio clínico aleatório. Journal of Science and Medicine in Sport. v. 04, n. 12. 2017 Disponível em:

<http://dx.doi.org/10.1016/j.jsams> Acesso em: 20 maio 2019. 
ANAIS DA VIII JORNADA DE FISIOTERAPIA DA CHRISFAPI, 2019;02-38

\title{
A INTERVENÇÃO DA FISIOTERAPIA NA QUALIDADE DE VIDA DE MULHERES \\ MASTECTOMIZADAS: revisão integrativa
}

\author{
Gylvana de Sousa Carvalho*1 , Káren Andresa Mendes da Silva ${ }^{1}$, Tásia Peixoto de Andrade \\ Ferreira $^{2}$ \\ 1 Instituição: Cristo Faculdade do Piauí - CHRISFAPI, \\ 2 Docente da Cristo Faculdade do Piauí - CHRISFAPI.
}

\section{RESUMO}

INTRODUÇÃO: O câncer de mama é o segundo tipo de neoplasia maligna mais presente entre as mulheres, podendo ser diagnosticado e tratado das mais variadas formas. Porém quando essa terapêutica envolve a mastectomia, reflete de forma negativa, tendo como consequência a perda da qualidade de vida. OBJETIVO: O estudo teve como objetivo mostrar como a intervenção da fisioterapia pode melhorar a qualidade de vida de mulheres mastectomizadas. METODOLOGIA: Foi realizada uma pesquisa nas por meio de artigos científicos, em bases de dados como SciELO, LILACS, Biblioteca Virtual de Saúde - BVS que abordasse sobre a intervenção fisioterapêutica na qualidade de vida de mulheres mastectomizadas. OS critérios de inclusão foram artigos que apresentem embasamento científico relacionado ao tema proposto, gratuitos e completos, que se enquadrassem nos anos de 2008 a 2019, publicados e indexados nos referidos bancos de dados, nos idiomas português e inglês. Como critérios de exclusão: artigos que não fossem gratuitos, textos incompletos, que não se enquadrassem dentro do período proposto e artigos que não retratassem a temática. RESULTADOS E DISCUSSÃO: A fisioterapia demonstrou eficácia e importância na prevenção e no tratamento de diversas complicações, que diminuem a qualidade de vida, como o linfedema, a diminuição da amplitude de movimento, dores neuropáticas, que são sequelas sofridas por mulheres mastectomizadas. Tendo assim, impacto positivo na qualidade de vida. CONCLUSÃO: Apesar disso, a fisioterapia pode recuperar essas desordens de forma não invasiva, repercutindo de forma positiva sobre todos os campos que englobam as alterações, levando assim, a uma melhora na qualidade de vida pós-mastectomia.

Palavras Chaves: Mastectomia. Qualidade de vida. Fisioterapia. 
ANAIS DA VIII JORNADA DE FISIOTERAPIA DA CHRISFAPI, 2019;02-38

\section{REFERÊNCIAS}

ALVES, Vanessa Lacerda. NETO, Miguel Sabino. ABLA, Luiz Eduardo Felipe. Avaliação precoce da qualidade de vida e autoestima de pacientes mastectomizadas submetidas ou não à reconstrução mamária. Disponível em:<

file://f:/artigo\%20de\%20ginaco/v32n2a09.pdf>. Acesso em: 2017.

CARVALHO, Francisco José O.V. CARDOSO, Maria dos Prazeres Carneiro. Eficácia das intervenções fisioterapêuticas aplicadas em mulheres. Disponível em:<

file://f:/artigo\%20de\%20ginaco/5\%20emana.\%20eficácia\%20das\%20intervenções\%20fisiot erapêuticas\%20aplicadas\%20em\%20mulheres\%20mastectomizadas\%20_\%20novafisio.pdf >. Acesso em: 2015.

CEZAR, Kaciani. NASCIMENTO, Alessandra Pinheiro Costa. Quality of Life of Breast Cancer Patients during Post-Treatment Period. Disponível em:<

file://e:/artigo\%20de\%20gineco/545-2090-1-pb.pdf>. Acesso em: 2014. 


\title{
ANAIS DA VIII JORNADA DE FISIOTERAPIA DA CHRISFAPI, 2019;02-38
}

\section{O USO DA ACUPUNTURA NO TRATAMENTO DA DEPRESSÃO:}

revisão integrativa

\begin{abstract}
Gylvana de Sousa Carvalho *1, Isac Leite Sousa1, Káren Andresa Mendes da Silva ${ }^{1}$, Manoel Wilson de Cerqueira Júnior ${ }^{1}$, lara Tamires Ibiapina Sousa de Castro²

1 Instituição: Cristo Faculdade do Piauí - CHRISFAPI,

2 Preceptora da Cristo Faculdade do Piauí - CHRISFAPI.
\end{abstract}

\section{RESUMO}

INTRODUÇÃO: A depressão tem sido caracterizada como episódio patológico no qual existe perda de interesse ou prazer, distúrbios do sono e apetite, retardo motor, sentimentos de inutilidade ou culpa, distúrbios cognitivos, diminuição da energia e pensamentos de morte ou suicídio. Dentre as práticas de terapias para o tratamento da Depressão, têm se destacado a acupuntura, que se demonstrou eficaz e engloba diferentes tipos de técnicas. OBJETIVO: avaliar as evidências científicas disponíveis na literatura sobre os efeitos da acupuntura no tratamento da depressão e a qualidade desses estudos. METODOLOGIA: trata-se de uma revisão integrativa, realizada nas bases/bancos de dados: Literatura Latino-Americana e do Caribe em Ciências da Saúde (LILACS); Medical Literature Analysis and Retrievial System Online (MEDLINE) e Scientific Electronic Library Online (SciELO), no mês de novembro de 2017. Os descritores depressão, acupuntura e terapia por acupuntura foram combinados entre si para garantir a ampla busca de estudos primários. RESULTADOS: dos 270 artigos, 8 foram selecionados para leitura na íntegra e 2 incluídos. Os resultados mostram efeitos positivos e estatisticamente significativos do uso da acupuntura para tratamento de indivíduos com depressão. CONCLUSÃO: a acupuntura parece ser um tratamento promissor para a depressão, no entanto, há necessidade de melhorar a qualidade metodológica das pesquisas nessa temática.

Palavras- chaves: Acupuntura. Depressão. Terapia por Acupuntura. 


\section{REFERÊNCIAS}

APÓSTOLO, J. L. A; Depressão, ansiedade e estresse em usuários de cuidados primários de saúde. Rev. Latino-Am. Enfermagem, 2011.

BRANDÃO, W. B.; MENEZES, C. R. O. M.; MOREIRA, A. C. P. Base Neurofisiológica para Compreensão da Dor através da Acupuntura. Rev. Dor, Vitória da Conquista, 11(2): 161168, 2010 . Disponível em: < http://files.bvs.br/upload/S/1806-0013/2010/v11n2/a1486.pdf >. Acesso em: 22 Nov 2017.

CASTRO, I. M. Auxílio da Acupuntura no Tratamento da Depressão. Facul. de Ciênc. da Ed. e Saúde, Brasília, 2011. Disponível em: < http://repositorio.uniceub.br/bitstream/123456789/2781/2/20625934.pdf>. Acesso em: 22 Nov 2017.

MENDES, K; SILVEIRA, R; GALVAO, C. Revisão integrativa: método de pesquisa para a incorporação de evidências na saúde e na enfermagem. Texto contexto - enferm., Florianópolis, v. 17, n. 4, Dec. 2008. 


\title{
ANAIS DA VIII JORNADA DE FISIOTERAPIA DA CHRISFAPI, 2019;02-38
}

\section{O USO DA ELETROESTIMULAÇÃO TRANSCUTÂNEA DO NERVO TIBIAL NO TRATAMENTO DE PACIENTES COM BEXIGA HIPERATIVA: revisão integrativa}

\author{
Taynara Lorrana Oliveira Araujoㄹ, Lyandra Ellen Bezerra Meneses ${ }^{1}$, Tásia Peixoto de \\ Andrade Ferreira ${ }^{2}$ \\ 1 Instituição: Cristo Faculdade do Piauí - CHRISFAPI \\ 2 Docente da Cristo Faculdade do Piauí - CHRISFAPI.
}

\section{RESUMO}

INTRODUÇÃO: A Bexiga Hiperativa $(\mathrm{BH})$ é considerada como uma patologia do trato urinário inferior, podendo estar relacionada a urgência miccional, o aumento da frequência urinária durante o dia e a noite, a mesma pode não está ligada a incontinência urinária. Essa patologia pode interferir bastante na qualidade de vida do individuo, pois ocasionará tristeza, frustação, baixa autoestima, vergonha, isolamentos e consequentemente uma perda de produtividade em suas atividades de vida diária. Pode-se destacar como principal sintoma nesta patologia a frequência urinária. Os dois principais tratamentos da $\mathrm{BH}$ são 0 medicamentoso e o fisioterapêutico. O tratamento medicamentoso consiste na ação dos anticolinérgicos sendo, porém, parcialmente eficazes em decorrência da sua baixa efetividade, além também dos seus efeitos adversos provocados. Dentre as principais formas de fisioterapia para o tratamento da $\mathrm{BH}$ são os exercícios do períneo para o ganho de força dos músculos do assoalho pélvico (MAP), a Eletroestimulação do Nervo Tibial Posterior (ENTP) e o tratamento comportamental, com objetivo de melhorar a qualidade de vida e reduzir os sintomas. OBJETIVO: Enfatizar os efeitos do uso da Eletroestimulação Transcutânea do Nervo Tibial no tratamento de pacientes com Bexiga Hiperativa. METODOLOGIA: O presente estudo trata-se de uma revisão integrativa de literatura. Foram usados artigos das bases: Medical Literature Analysis and Retrieval System Online (Medline), Literatura Latino-Americana e do Caribe em Ciências da Saúde (LILACS) e Scientific Eletronic Library Online (SciELO). Como critérios de inclusão, foram utilizados estudos que compreendessem os últimos 10 anos (2012 a 2019); textos completos na íntegra; que fossem ensaios clínicos randomizados; estudos controles; relatos de casos, estudo piloto ;publicados nas língua portuguesa, espanhola, inglesa; que apresentassem Quális Periódico (A1 a B2) e que tivessem como recurso de tratamento fisioterapêutico a eletroestimulação do nervo tibial em pacientes com diagnóstico de bexiga hiperativa. 
ANAIS DA VIII JORNADA DE FISIOTERAPIA DA CHRISFAPI, 2019;02-38

Excluindo aqueles que estivessem incompletos; artigos repetidos; artigos que não tratassem sobre o assunto; amostra inadequada e publicações inferiores ao ano de 2012. RESULTADOS E DISCUSSÃO: Evidenciaram-se efeitos benéficos da ENTP em pacientes com bexiga hiperativa na maioria dos estudos analisados. Além disso, os resultados negativos obtidos da ENTP em uma das pesquisas utilizadas, pode ser explicado pela idade dos pacientes contidos na amostra, pois o tratamento urológinecológico com crianças devido ao seu sistema fisiológico como também seu comportamento durante a terapia, possui algumas dificuldades na resolução dos achados. CONCLUSÂO: No presente artigo pode-se observar vários efeitos benéficos nos estudos mencionados. Os objetivos dessa pesquisa foram alcançados, porém ainda não há evidências suficientes que comprovem que a eletroestimulação transcutânea do nervo tibial é o melhor tratamento para Bexiga Hiperativa, dessa maneira a junção com outras terapias alternativas estabelece um melhor prognóstico para o paciente. Todavia, é necessário mais pesquisas aprofundadas a respeito desse tema.

Palavras-chaves: Eletroestimulação. Bexiga. Hiperativa. Fisioterapia.

\section{REFERÊNCIAS}

ARRUDA, Raquel Martins et.al. Hiperatividade do detrusor: comparação entre oxibutinina, eletroestimulação funcional do assoalho pélvico e exercícios perineais. Estudo Randomizado. Revista Brasileira de Ginecologia Obstetrícia, v.29,n.9,p.452-8,2007.

JR, Ubirajara Barroso et.al. Posterior Tibial Nerve Stimulation vs Parasacral Transcutaneous Neuromodulation for Overactive Baldder in Children. The Journal of Urology, v.190, p.637-677, August 2013.

MAGALDI, Cristiane Milani et.al.Efeito da Eletroestimulação do Nervo Tibial em indivíduos portadores de bexiga neurogênica. Fisioscience, 2013.

RUFINO, Priscila Thaís Santos de Oliveira, LEME,Ana Paula Cardoso Batista Paes. Efeito da eletroestimulaçao do nervo tibial posterior para bexiga hiperativa em mulheres: revisão sistemática. Revista Pesquisa Fisioterapia, v.8 ,n.3,p.420-429, Agosto 2018. 
ANAIS DA VIII JORNADA DE FISIOTERAPIA DA CHRISFAPI, 2019;02-38

\title{
ANÁLISE DA QUALIDADE DE VIDA E DA CAPACIDADE FUNCIONAL DE INDIVÍDUOS COM DOR LOMBAR CRÔNICA
}

\author{
Carolyne Carvalho Caxias"; Mayane Carneiro Alves Pereira² \\ 1 Graduanda do Curso de Fisioterapia pela Cristo Faculdade do Piauí - CHRISFAPI \\ 2 Docente do Curso de Fisioterapia pela Cristo Faculdade do Piauí - CHRISFAPI
}

\section{RESUMO}

INTRODUÇÃO: Um dos principais problemas da população brasileira, principalmente em idade produtiva são as doenças crônicas que afetam a condição musculoesquelética. Entre essas doenças, destacam-se os problemas crônicos de coluna, como dores lombares e os distúrbios osteomusculares relacionados ao trabalho (DORT). OBJETIVO: O objetivo desse estudo é analisar a qualidade de vida e a capacidade funcional de indivíduos com dor lombar crônica. METODOLOGIA: Trata-se de um estudo transversal descritivo, realizado com 11 indivíduos portadores de dor lombar crônica, atendidos no Centro de Reabilitação Municipal de Piripiri-PI. Para a coleta de dados foi realizada a aferição da pressão arterial (P.A), o preenchimento de um formulário que abrange aspectos sociodemográficos, a Escala Visual Analógica de Dor (EVA), bem como o Questionário Genérico de Avaliação de Qualidade de Vida SF-36 (Short Form-36), Questionário de Desempenho Funcional de Roland Morris e Teste de Capacidade Física (sentado para de pé). RESULTADOS E DISCUSSÃO: Verificouse que a média de idade dos indivíduos foi de 55,4 ( $\pm 10,7)$ anos, sendo a maioria pertencente ao sexo feminino, possuindo apenas o ensino fundamental. Na avaliação da dor, foi observada uma média de 5,0 sendo considerada dor moderada. Ao analisar os resultados do questionário de Roland Morris, verificou-se que $81,8 \%$ dos indivíduos foram considerados incapacitados funcionalmente devido à lombalgia crônica. Em relação ao teste de capacidade física (sentado para de pé) o tempo médio de realização foi de 0,55 segundos. Já no questionário Genérico de Avaliação de Qualidade de Vida SF-36 observou-se que todos os indivíduos obtiveram escores médios menor que 50,0 pontos exceto na variável de "saúde mental" obteve 56,0 pontos. Evidencia-se o valor observado na categoria "aspectos físicos" de 2,3 pontos, próximo a 0. CONCLUSÃO: Os resultados indicam a necessidade de serem utilizados instrumentos que avaliem os aspectos de qualidade de vida e capacidade funcional em pacientes com lombalgia crônica de modo que contribua para o melhor direcionamento nos programas de reabilitação. 
ANAIS DA VIII JORNADA DE FISIOTERAPIA DA CHRISFAPI, 2019;02-38

Palavras-Chave: Qualidade de vida. Capacidade funcional. Dor lombar crônica.

\section{REFERÊNCIAS}

MACEDO, C. S. G.; DEBIAGI, P. C.; ANDRADE, F. M. Efeito do Isostretching na resistência muscular de abdominais, glúteo máximo e extensores de tronco, incapacidade e dor em pacientes com lombalgia. Fisioterapia em Movimento. Curitiba, v. 23, n.1, p. 113-120, jan./mar. 2010.

MASCARENHAS, Claudio Henrique Meira; SANTOS, Leandro Silva. Avaliação da dor e da capacidade funcional em indivíduos com lombalgia crônica. Journal of the Health Sciences Institute. V. 29, n. 3, p. 205-208, 2011.

OLIVEIRA, M. M.; ANDRADE, S. S. C. A.; SOUZA, C. A. V. et al. Problema crônico e diagnóstico de distúrbios osteomusculares relacionados ao trabalho (DORT) autorreferidos no Brasil: Pesquisa Nacional de Saúde, 2013. Epidemiologia e Serviços de Saúde. Brasília, v. 24, n. 2, p. 287-296, abr./jun. 2015.

RODRIGUES, C. P.; SILVA, R. A.; NETO, E. N et al. Análise da capacidade funcional em indivíduos com e sem dor lombar crônica. Acta Ortopédica Brasileira. São Paulo, v. 25, n. 4, jul./ago. 2017.

STEFANE, T.; SANTOS, A. M.; MARINOVIC, A.; HORTENSE, P. Lombalgia crônica: intensidade da dor, deficiência e qualidade de vida. Acta Paulista de Enfermagem. V. 26, n. 1, p. 14-20, 2013. 


\section{ANAIS DA VIII JORNADA DE FISIOTERAPIA DA CHRISFAPI, 2019;02-38}

TUBERCULOSE: uma abordagem geral dos principais aspectos

Élita Sampaio Emiliano1; Naiane da Silva Cruz¹; Tânia Valéria Marques Brito1.

${ }^{1}$ Discente da Cristo Faculdade do Piauí - CHRISFAPI, Piripiri

\section{RESUMO}

INTRODUÇÃO: O termo tuberculose se deve pelo fato da doença provocar lesões chamadas de tubérculos, causada por bactéria do gênero Mycobacterium classificada como Mycobacterium tuberculosis, também denominado de Bacilo de Koch que são bastonetes delgados aeróbicos com crescimento em cadeias retas ou ramificadas de parede celular cerácea única com ácido micólico e fracamente Gram-positivas. A maioria dos casos de tuberculose é de responsabilidade deste microrganismo, mas há também a tuberculose orofaríngea e intestinal, contraída através da ingestão do leite contaminado, considerada rara em locais onde há esterilização do alimento (ROBBINS e COTRAN, 2010; MELO et al. 2012).Segundo a Organização Mundial de Saúde (OMS), o Brasil apresenta 46 mil casos novos em 100 mil habitantes por ano (WHO, 2008). A doença apresenta algumas características marcantes como: um longo período de latência entre a infecção inicial e a apresentação clínica da doença; preferência pelos pulmões, mas também pode ocorrer em outros órgãos do corpo como ossos, rins e meninges; e resposta granulomatosa associada à intensa inflamação e lesão tissular (Iseman, 2005). A tuberculose pode ser classificada como primária, secundária, endobrônquica, endotraqueal e laríngea, progressiva, miliar sistêmica e isolada (ROBBINS e COTRAN, 2010; MURTA, 2012; MELO et al. 2012). METODOLOGIA: Trata-se de estudo de revisão integrativa de literatura, pesquisa qualitativa, análise de literatura científica, artigo de revisão. Para a identificação de produções sobre o tema, entre anos 2005 e 2012. Visando a compreensão de determinado tema, a partir de outros estudos independentes. Para o levantamento dos artigos na literatura, realizou-se uma busca nas seguintes bases de dados: Literatura Latino-Americana e do Caribe em Ciências da Saúde (LILACS) e Medical Literature Analysis and Retrieval Sistem on-line (Medline) Scielo, onde foram escolhidos cinco artigos que abrangeu a TB com suas características. Foram empregados, para busca dos artigos, os seguintes descritores e suas combinações nas línguas portuguesa: tuberculose geral, tratamento, buscando-se artigos em português, com data de publicação posterior ao ano de 2005. RESULTADOS E DISCUSSÃO: Pela análise dos textos escolhidos, os cinco artigos estudados para o presente trabalho foram 
ANAIS DA VIII JORNADA DE FISIOTERAPIA DA CHRISFAPI, 2019;02-38

classificados como estudo de caso, revisão integrativa de literatura, pesquisa qualitativa, análise de literatura científica, artigo de revisão, livro e site. De acordo com os artigos publicados, a TB ainda é uma doença negligenciada pela população brasileira. Fazendo com que a doença ainda continue crescendo e haja mais pacientes com abandono de tratamento, levando com que a incidência da tuberculose e seu contágio continue aumentando com o passar dos anos. CONCLUSÃO: Concluiu-se que esse estudo permitiu verificar que mesmo a TB sendo uma doença antiga e conhecida pelo mundo, ainda há um certo espaço do desconhecido e de não controle na sociedade. Mostrando que ainda há muito o que se construir no controle e tratamento, buscando formas de diminuição de abandono dos pacientes tuberculosos e aumento de conhecimento sobre sua prevenção. A realização de visitas domiciliares é um desafio às equipes de saúde. Além do empenho destas, é necessário que a gestão forneça subsídios, como qualificação profissional, materiais e tempo para sua implantação, o que acarretará em melhora dos indicadores de acesso ao paciente. É importante também que o paciente conclua o tratamento.

Palavra-chaves: Tuberculose. Tratamento. Tratamento.

\section{REFERÊNCIAS}

FURLAN, Mara Cristina Ribeiro; MARCON, Sonia Silva. Avaliação do acesso ao tratamento de tuberculose sob a perspectiva de usuários. Cad. saúde colet., Rio de Janeiro, v. 25, n. 3, p. 339-347, July 2017.

ROBBINS \&COTRAN. Patologia: bases patológicas das doenças. Editora Saunders Elsevier Ltda. 8e․ 2010. Rio de janeiro, RJ.

NOGUEIRA, A. F.; FACCHINETTI, V.; SOUZA, M. V.N.; Vasconcelos, T. R. A. Tuberculose: uma abordagem geral dos principais aspectos. Revista Brasileira de Farmácia. Rio de janeiro; 2010.

MARQUES, Caroline Fonseca. As percepções dos pacientes em tratamento de tuberculose sobre sua doença: uma análise da literatura científica.; Fundação Oswaldo Cruz. Rio de Janeiro; 2011.

RECH; Viviane Viegas; BERVIG, Daisy; RODRIGUES, Lenice Ferreira; SANCHES, Cristiano; FROTA, Roberto; Efeitos de um programa de exercícios físicos na tolerância ao esforço de indivíduos com tuberculose pulmonar, 2005.

CHIRINOS, Narda Estela Calsin; MEIRELLES, Betina Hörner Schlindwein; Fatores associados ao abandono do tratamento da tuberculose: uma revisão integrativa, 2011. 
ANAIS DA VIII JORNADA DE FISIOTERAPIA DA CHRISFAPI, 2019;02-38

https://minutosaudavel.com.br/o-que-e-tuberculose-sintomas-tratamento-e-tipos/ > acessado dia 07-05-2019, às 18:50 horário de Brasília. 


\title{
A UTILIZAÇÃO DA VENTOSATERAPIA NA REDUÇÃO DO FIBRO EDEMA GELÓIDE - TEORIA CIRCULATÓRIA
}

\author{
Amanda Ferreira Alves ${ }^{1}$; Andrea da Siva Feitosa1; Daiany de Sousa Monteiro² \\ ${ }^{1}$ Discente da Cristo Faculdade do Piauí - CHRISFAPI, Piripiri \\ 2 Docente da Cristo Faculdade do Piauí - CHRISFAPI, Piripiri
}

\section{RESUMO}

INTRODUÇÃO: A patologia acometida nas mulheres denominada Fibro Edema Gelóide (celulite) se trata de uma disfunção localizada que afeta o tecido dérmico e subcutâneo, com alterações vasculares e lipodistrofia, a Teoria Circulatória é caracterizada como causa para o déficit circulatório, impedindo assim a livre circulação dos líquidos intersticiais. Objetivo: Melhorar a oxigenação e ativar a circulação local através da Ventosaterapia. No intuito de comprovar sua eficácia no Fibro Edema Gelóide. METODOLOGIA: Este é um estudo de campo, 20 mulheres, entre 17 a 35 anos, realizada na clínica escola da Christus Faculdade do Piauí - Chrisfapi, situada na cidade de Piripiri, Piauí. Serão 10 atendimentos de 10 minutos, com registros fotográficos do aparelho Celluvision na primeira, na quinta e na última sessão de cada paciente, registrando o local que será realizado o procedimento, para demonstração dos resultados. RESULTADOS: O estudo só teve início após a provação do Comitê de Ética em Pesquisa (CEP) com os seguintes dados do parecer de aprovação com o número do parecer: 2.868.077, data da relatoria: 02/09/2018. Foram avaliadas 30 mulheres, todas apresentaram FEG de graus I, II á III, entre tanto, nove mulheres eram de grau II e III na qual foram diretamente incluídas no estudo. As outras pacientes tinham o mesmo grau de fibro edema gelóide, portanto foram sorteadas as outras onze pacientes para o estudo, completando o total de 20 pacientes. Após os dez atendimentos das 20 pacientes, e os registros fotográficos das mesmas foi possível observar um grande resultado nos três tipos de FEg, mas os graus I e II evidentemente obtiveram melhores resultados, pois não são graus tão acometidos como o grau III. CONCLUSÃO: Com o presente estudo foi possível concluir que a Ventosaterapia é muito eficas na redução do FEG, melhorando a visão corporal das mulheres e proporcionando efeitos fisiológicos agradáveis para as mesmas, reduzindo o edema, as dores e o encomodo visual do FEG. Logo, é possível concluir que ateoria circulatória está diretamente ligada ao FEG, e com a técnica de Ventosaterapia é 
ANAIS DA VIII JORNADA DE FISIOTERAPIA DA CHRISFAPI, 2019;02-38

possível reduzi-la, assim, melhorando a circulação local e reduzindo a FEG.

Palavras-chave: Fibro edema gelóide. Ventosaterapia. Termografia.

\section{REFERÊNCIAS}

MEYER, Patrícia Froes. Desenvolvimento e aplicação de um protocolo de avaliação fisioterapêutico em pacientes com fibro edema gelóide. Curitiba, 2005.

GUIRRO, Elaine.; GUIRRO, Rinaldo. Fisioterapia Dermato-Funcional: Fundamentos Recursos Patologias. o ed. Revisada e ampliada, 2004.

BORGES, Fábio D. S. Dermato Funcional: Modalidades Terapêuticas nas Disfunções Estéticas. $2^{\circ}$ ed. Revisada e ampliada, 2010.V 


\section{A ATUAÇÃO DA FISIOTERAPIA EM MULHERES COM DISMENORREIA PRIMÁRIA:}

revisão integrativa

Carla Maysla Oliveira Brito ${ }^{1}$, Graciele Gomes Damasceno ${ }^{1}$, Tásia Peixoto de Andrade Ferreira ${ }^{2}$

1 Instituição: Cristo Faculdade do Piauí - CHRISFAPI,

2 Docente da Cristo Faculdade do Piauí - CHRISFAPI.

\section{RESUMO}

INTRODUÇÃO: A dismenorreia é uma desordem ginecológica que atinge até 60\% das mulheres, podendo ser classificada em primária ou secundária. A fisioterapia voltada para o alívio da dor pélvica está baseada em produzir mudanças físicas na musculatura lisa/estriada e sistema nervoso central. OBJETIVO: O presente estudo tem como objetivo verificar a eficácia da atuação fisioterapêutica em mulheres com dismenorreia primária. METODOLOGIA: A pesquisa trata-se de uma revisão integrativa. Para a busca de artigos utilizou-se a Biblioteca Virtual de Saúde (BVS) e PubMed por meio das bases de dados indexadas: LILACs, MEDLINE e SCIELO. Foram incluídos artigos na língua portuguesa e inglesa, completos, gratuitos e publicados nos últimos 10 anos; e excluídos os que se apresentassem fora do período demarcado, revisões de literatura e conclusões de curso. RESULTADOS: As participantes relataram redução no quadro de algia e diminuição no número de comprimidos utilizados para a dor. CONCLUSÃO: Visto isso, conclui-se que a fisioterapia tem uma eficácia na dismenorreia primária, podendo ser considerada uma opção segura, não farmacologia e não invasiva no tratamento.

Palavras-chave: Fisioterapia. Dismenorreia. Saúde da Mulher.

\section{REFERÊNCIAS}

BAI, Han-Yan et al. Effect of transcutaneous electrical nerve stimulation therapy for the treatment of primary dysmenorrheal. General Medicine Open. 2017; 96:36 (e7959)

BARACHO, Elza. Fisioterapia Aplicada à Saúde da Mulher. 5a ed. Rio de Janeiro: Guanabara Koogan, 2014.

BEREK, Jonathan S.; NOVAK, Edmund R. Tratado de Ginecologia. 15aㅡ ed. Rio de Janeiro: 
Guanabara Koogan, 2014.

BLÖDT, Susanne et al. Effectiveness of app-based self-acupressure for women with menstrual pain compared to usual care: a randomized pragmatic trial. American Journal of Obstetrics and Gynecology. 2018; 218: 227.e1-9.

GEBER, Selmo et al. Guia de Bolso de Ginecologia. $1^{\text {a }}$ ed. São Paulo: Editora Atheneu, 2013.LIMA, Geraldo Rodrigues de. Ginecologia Clínica. $1^{1}{ }^{a}$ ed. São Paulo: Editora Atheneu, 2015.

MENDES, Karina Dal Sasso et al. Revisão Integrativa: método de pesquisa para a incorporação de evidências na saúde e na enfermagem. Texto Contexto Enferm. Florianópolis, 2008 Out-Dez.

REIS, Conceição Aparecida de Almeida Santos et al. The effectiveness of connective tissue massage in the treatment of primary dysmenorrhea among young women. Rev. Bras.

Saude Mater. Infant. vol.10 no.2 Recife Apr./June 2010.

RODRÍGUEZ, María Isabel Tomás et al. Effectiveness of medical taping concept in primary dysmenorrhoea: a two-armed randomized trial. Scientific Reports. 2015.

STALLBAUM, Joana Hasenack et al. Efeitos da bandagem funcional elástica sobre a dismenorreia primaria em universitárias. Fisioterapia Brasil. 2016; 17(6): 518-525

STEPHENSON, Rebecca G.; O'CONNOR, Linda J. Fisioterapia Aplicada à Ginecologia e Obstetrícia. $2^{\mathrm{a}}$ ed. São Paulo: Manole, 2004. 